\title{
A Mild, Chemoselective Oxidation of Sulfides to Sulfoxides Using $o$-Iodoxybenzoic Acid (IBX) and Tetraethylammonium Bromide as Catalyst.
}

Vidyanand G. Shukla, Paresh D. Salgaonkar \& Krishnacharya G. Akamanchi ${ }^{*}$

\begin{tabular}{|c|c|c|}
\hline Sr. No. & Content & Page Numbers \\
\hline 1. & $\begin{array}{c}\text { General Methods for Preparation of Sulfides With } \\
\text { Characterization }\end{array}$ & S2-S5 \\
\hline 2. & $\begin{array}{c}\text { General Method for Preparation of Sulfoxides } \\
\text { with Characterization }\end{array}$ & S6-S9 \\
\hline 3. & References & S9-S10 \\
\hline
\end{tabular}




\section{Part I: Preparation of Sulfides:}

The corresponding sulfide substrates (Entry 1-3, Table 2) were procured from commercial sources and used directly.

\section{Preparation of Sulfides (Entry 4, 5, Table 2)}

Method A: $\quad$ Aromatic thiol (1.0 eq), sodium hydroxide (1.6 eq), alkyl halide (MeI) (1.1 eq) in benzene : water (1:1) and cetyltrimethylammonium bromide $(\sim 0.1 \mathrm{eq})$ were combined and stirred vigorously at ambient temperature. The reaction was monitored by TLC. On completion of the reaction, the organic layer was separated, washed with water, dried over sodium sulfate and evaporated on a rotary evaporator to afford the corresponding sulfides. Purification on silica gel column chromatography afforded the pure sulfides. The compounds were in confirmation with those reported in the literature. ${ }^{1}$

\section{Preparation of p-(1-methoxyethyl)phenyl methyl sulfide (Entry 6, Table 2)}

Method B: To a solution of p-methylthioacetophenone $(0.23 \mathrm{~g}, 1.386 \mathrm{mmol})$ in $10 \mathrm{ml}$ of methanol was added sodium borohyride $(0.06 \mathrm{~g}, 1.524 \mathrm{mmol})$. The reaction mixture was stirred for $30 \mathrm{~min}$ at room temperature. On completion of the reaction, $15 \mathrm{ml}$ of $10 \%$ hydrochloric acid was added and stirred for 30 minutes. The reaction mass was extracted with ethyl acetate. Organic layer washed thoroughly with water, dried over sodium sulfate and concentrated in vacuo to yield the sulfide. It was further purified by silica gel column chromatography using EtOAc: Hexane (1:9 v/v).

IR $\left(\mathrm{CHCl}_{3}, \mathrm{~cm}^{-1}\right): v 3005.9,1216.8,1102.5,748.8 .{ }^{1} \mathrm{H}$ NMR: $\delta 1.226(\mathrm{~d}, \mathrm{~J}=6.50 \mathrm{~Hz}$, 3H), $2.366(\mathrm{~s}, 3 \mathrm{H}), 3.066(\mathrm{~s}, 3 \mathrm{H}), 3.929(\mathrm{q}, \mathrm{J}=6.50 \mathrm{~Hz}, 1 \mathrm{H}), 7.089-7.199(\mathrm{~m}, 4 \mathrm{H})$ p-Methylthioacetophenone (Entry 7, Table 2) was prepared by literature procedure. ${ }^{2}$ Preparation of Sulfides (Entry 8-12, Table 2) 
All these sulfides were prepared by following Method $\mathrm{A}$ and using $\mathrm{EtBr}$ as alkylating agent. The compounds were in confirmation with reported literature data. ${ }^{3}$

\section{Preparation of Sulfides (Entry 13-15, Table 2)}

Method C: To a solution of $\alpha, \beta$-unsaturated compound (acrylonitirle, ethyl acrylate, methyl crotonate) $(1.0 \mathrm{eq})$ in benzene was added thiophenol (1.1 eq) followed by triethylamine (1.0 eq). The reaction was stirred at room temperature and monitored by TLC. On completion of the reaction, the organic layer was separated and washed with $10 \%$ hydrochloric acid ( 3 X $15 \mathrm{ml})$, water $(2 \times 10 \mathrm{ml}), 5 \% \mathrm{NaOH}(2 \times 15 \mathrm{ml})$, and water (2 X $10 \mathrm{ml})$. The organic layer was dried over sodium sulphate and concentrated in vacuo to yield the sulfide. Further purification on silica gel column chromatography gave the pure sulfides.

\section{2-Cyanoethyl phenyl sulfide (Entry 13, Table 2)}

${ }^{1} \mathrm{H}$ NMR: $\delta 2.305(\mathrm{t}, \mathrm{J}=6.60 \mathrm{~Hz}, 2 \mathrm{H}), 2.985(\mathrm{t}, \mathrm{J}=6.60 \mathrm{~Hz}, 2 \mathrm{H}), 7.114-7.260(\mathrm{~m}, 5 \mathrm{H})$.

\section{2-Ethoxycarbonylethyl phenyl sulfide (Entry 14, Table 2)}

${ }^{1} \mathrm{H}$ NMR: $\delta 0.961(\mathrm{t}, \mathrm{J}=6.90 \mathrm{~Hz}, 3 \mathrm{H}), 2.195(\mathrm{t}, \mathrm{J}=7.08 \mathrm{~Hz}, 2 \mathrm{H}), 2.871(\mathrm{t}, \mathrm{J}=7.08 \mathrm{~Hz}$, 2H), $3.775(\mathrm{q}, \mathrm{J}=6.90 \mathrm{~Hz}, 2 \mathrm{H}), 7.020-7.134(\mathrm{~m}, 5 \mathrm{H})$.

\section{2-Methoxycarbonyl-1-methylethyl phenyl sulfide (Entry 15, Table 2)}

${ }^{1} \mathrm{H}$ NMR: $\delta 1.460(\mathrm{~d}, \mathrm{~J}=6.70 \mathrm{~Hz}, 3 \mathrm{H}), 2.553(\mathrm{t}, \mathrm{J}=9 \mathrm{~Hz}, 2 \mathrm{H}), 3.600-3.800(\mathrm{~m}, 4 \mathrm{H})$, 7.300-7.510 (m, 5H).

\section{Preparation of 2-Hydroxycarbonyl-1-methylethyl phenyl sulfide (Entry 16, Table 2)}

Method D: A mixture of 2-Methoxycarbonyl-1-methylethyl phenyl sulfide (Entry 15, Table 2) in 50\% hydrochloric acid was refluxed with vigorous stirring for $6 \mathrm{~h}$. The reaction was allowed to cool to room temperature and was extracted with chloroform. 
The organic layer was washed successively with water and dried over sodium sulphate. Concentration in vacuo afforded the sulfide that was further purified by silica gel column chromatography.

${ }^{1} \mathrm{H}$ NMR: $\delta 1.120(\mathrm{~d}, \mathrm{~J}=6.60 \mathrm{~Hz}, 3 \mathrm{H}), 2.120-3.623(\mathrm{~m}, 3 \mathrm{H}), 7.190-7.470(\mathrm{~m}, 5 \mathrm{H})$.

\section{Preparation of Sulfides (Entry 17, 18, Table 2)}

Method E:

\section{A) Michael Addition:}

To a solution of crotonaldehyde (1.0 eq) in benzene was added aromatic thiol (thiophenol/ p-chlorothiophenol) (1.1 eq) followed by triethylamine $(1.0 \mathrm{eq})$. The reaction mixture was stirred at room temperature and monitored by TLC. On completion of the reaction, organic layer washed successively with $10 \%$ hydrochloric acid $(3 \mathrm{X} 15$ $\mathrm{ml}$ ), water (2X $10 \mathrm{ml}), 5 \%$ sodium hydroxide ( 2 X $15 \mathrm{ml})$, and water ( 2 X10 ml). Organic layer dried over sodium sulphate and concentrated in vacuo to yield the corresponding sulfide Michael adducts.

\section{3-(Phenylthio)butanal}

${ }^{1} \mathrm{H}$ NMR: $\delta 1.258(\mathrm{~d}, \mathrm{~J}=6.60 \mathrm{~Hz}, 3 \mathrm{H}), 2.321(\mathrm{~d}, \mathrm{~J}=7.08 \mathrm{~Hz}, 2 \mathrm{H}), 3.351(\mathrm{~m}, 1 \mathrm{H}), 7.150-$ $7.415(\mathrm{~m}, 5 \mathrm{H}),, 9.675(\mathrm{~s}, 1 \mathrm{H})$.

\section{3-(p-Chlorophenylthio)butanal}

${ }^{1} \mathrm{H}$ NMR: $\delta 1.347(\mathrm{~d}, \mathrm{~J}=6.6 \mathrm{~Hz}, 3 \mathrm{H}), 2.431(\mathrm{~d}, \mathrm{~J}=7.32 \mathrm{~Hz}, 2 \mathrm{H}), 3.370(\mathrm{~m}, 1 \mathrm{H}), 7.210-$ $7.560(\mathrm{~m}, 4 \mathrm{H}), 9.790(\mathrm{~s}, 1 \mathrm{H})$.

\section{B) Reduction:}

The corresponding Michael adduct sulfides from Step A above (1.0 eq) were dissolved in methanol and sodium borohydride (1.25 eq) was added in portions. Reaction 
mixture was stirred at room temperature for 30 minutes. On completion of the reaction, $10 \%$ hydrochloric acid was added and reaction mixture was extracted with ethyl acetate. Organic layer washed with water $(2 \mathrm{x} 10 \mathrm{ml})$ and dried over sodium sulphate. Concentration in vасио followed by purification over silica gel column chromatography afforded the pure sulfides.

\section{3-(Phenylthio)butan-1-ol (Entry 17, Table 2)}

${ }^{1} \mathrm{H}$ NMR: $\delta 1.222(\mathrm{~d}, \mathrm{~J}=6.65 \mathrm{~Hz}, 3 \mathrm{H}), 1.568(\mathrm{~m}, 2 \mathrm{H}), 2.386(\mathrm{~s}, 1 \mathrm{H}), 3.172-3.803(\mathrm{~m}$, 3H), 7.118-7.289 (m, 5H).

\section{3-(p-Chlorophenylthio)butan-1-ol (Entry 18, Table 2)}

${ }^{1} \mathrm{H}$ NMR: $\delta 1.279(\mathrm{~d}, \mathrm{~J}=6.65 \mathrm{~Hz}, 3 \mathrm{H}), 1.891(\mathrm{~m}, 2 \mathrm{H}), 2.61(\mathrm{~s}, 1 \mathrm{H}), 3.210-3.930(\mathrm{~m}, 3 \mathrm{H})$, 7.230-7.510 (m, 4H).

\section{Preparation of Sulfides (Entry 19, 20, Table 2)}

These were prepared as described in Method A (aryl/arylalkyl halides used were bromobenzene and benzyl chloride). The compounds were in accordance with the literature data. ${ }^{4}$ 


\section{Part II: Sulfoxidation}

General procedure: To a stirred suspension of IBX (1.1 eq) in $10 \mathrm{ml}$ of $\mathrm{CHCl}_{3}: \mathrm{H}_{2} \mathrm{O}$ (100:1) mixture was added tetraethylammonium bromide(TEAB) $(5 \%)$ followed by addition of sulfide $(1.0 \mathrm{eq})$ in one portion. The mixture was stirred at room temperature until complete consumption of starting material as observed by TLC. The residual solids were filtered off and washed thoroughly with $\mathrm{CHCl}_{3}$. The combined filtrate was washed successively with $10 \%$ sodium bisulfite solution ( 2 X $15 \mathrm{ml}$ ), saturated sodium bicarbonate solution ( 2 X $15 \mathrm{ml})$, water ( 2 X $15 \mathrm{ml})$, and brine (1 X $15 \mathrm{ml})$. The organic layer was dried over sodium sulfate and concentrated in vacuo. Purification by silica gel column chromatography (50\% EtOAc/hexane) afforded the corresponding sulfoxide.

Following the general procedure various sulfides were oxidized to afford sulfoxides (Entry 1-20, Table 2)

\section{Diethyl sulfoxide (Entry 1, Table 2)}

IR $\left(\mathrm{CHCl}_{3}, \mathrm{~cm}^{-1}\right): \vee 2915,1049$.

\section{Dibutyl sulfoxide (Entry 2, Table 2)}

IR $\left(\mathrm{CHCl}_{3}, \mathrm{~cm}^{-1}\right): v$ 2920, 1037. ${ }^{1} \mathrm{H}$ NMR: $\delta 0.993(\mathrm{t}, \mathrm{J}=6.12 \mathrm{~Hz}, 3 \mathrm{H}), 1.230-1.955(\mathrm{~m}$, $8 \mathrm{H}), 2.67(\mathrm{t}, \mathrm{J}=7.08 \mathrm{~Hz}, 4 \mathrm{H})$.

\section{Phenyl methyl sulfoxide (Entry 3, Table 2)}

IR $\left(\mathrm{CHCl}_{3}, \mathrm{~cm}^{-1}\right): v$ 3013.2, 1041, 749.7. ${ }^{1} \mathrm{H}$ NMR: $\delta 2.73(\mathrm{~s}, 3 \mathrm{H}), 7.45-7.75(\mathrm{~m}, 5 \mathrm{H})$. 


\section{p-Tolyl methyl sulfoxide (Entry 4, Table 2)}

IR $\left(\mathrm{CHCl}_{3}, \mathrm{~cm}^{-1}\right): v 2998.1,1249.3,1046.4,763.7 .{ }^{1} \mathrm{H}$ NMR: $\delta 2.43(\mathrm{~s}, 3 \mathrm{H}), 2.72(\mathrm{~s}, 3 \mathrm{H})$, $7.40(\mathrm{~d}, \mathrm{~J}=8.52 \mathrm{~Hz}, 2 \mathrm{H}), 7.66(\mathrm{~d}, \mathrm{~J}=8.52 \mathrm{~Hz}, 2 \mathrm{H})$

\section{p-Chlorophenyl methyl sulfoxide (Entry 5, Table 2)}

IR $\left(\mathrm{CHCl}_{3}, \mathrm{~cm}^{-1}\right): v$ 3003.1, 1042.3, 741.3. ${ }^{1} \mathrm{H}$ NMR: $\delta 2.618(\mathrm{~s}, 3 \mathrm{H}), 7.346-7.513(\mathrm{~m}$, $4 \mathrm{H})$

p-(1-Methoxyethyl)phenyl methyl sulfoxide (Entry 6, Table 2)

IR $\left(\mathrm{CHCl}_{3}, \mathrm{~cm}^{-1}\right): v 3004.2,1215,1044.7,751.9 .{ }^{1} \mathrm{H} \mathrm{NMR}: \delta 1.327(\mathrm{~d}, \mathrm{~J}=6.50 \mathrm{~Hz}, 3 \mathrm{H})$, $2.618(\mathrm{~s}, 3 \mathrm{H}), 3.184(\mathrm{~s}, 3 \mathrm{H}), 4.109$ (q, J=6.50 Hz, 1H), 7.309-7.635 (m, 4H)

p-Acetylphenyl methyl sulfoxide (Entry 7, Table 2)

m.p: $110^{0} \mathrm{C} . \mathrm{IR}\left(\mathrm{KBr}, \mathrm{cm}^{-1}\right): v \quad 3020.9,1690.7,1259.3,1046.7,766.1 .{ }^{1} \mathrm{H}$ NMR: $\delta 2.569$ (s, 3H), 2.692 (s, 3H), 7.598 (d, J= 8.52 Hz, 2H), 7.973 (d, J=8.52 Hz, 2H). MS (EI): 182 $\left(\mathrm{M}^{+}\right)$

\section{Phenyl ethyl sulfoxide (Entry 8, Table 2)}

IR $\left(\mathrm{CHCl}_{3}, \mathrm{~cm}^{-1}\right): v$ 3011.8, 1042.5, 753.6. ${ }^{1} \mathrm{H}$ NMR: $\delta 0.96(\mathrm{t}, \mathrm{J}=7.32 \mathrm{~Hz}, 3 \mathrm{H}), 2.47-$ $2.89(\mathrm{~m}, 2 \mathrm{H}), 7.05-7.43(\mathrm{~m}, 5 \mathrm{H})$

\section{p-Tolyl ethyl sulfoxide (Entry 9, Table 2)}

IR $\left(\mathrm{CHCl}_{3}, \mathrm{~cm}^{-1}\right): v 2993,1251.9,1043.7,771.7 .{ }^{1} \mathrm{H} \mathrm{NMR}: \delta 0.973(\mathrm{t}, \mathrm{J}=7.56 \mathrm{~Hz}, 3 \mathrm{H})$, $2.329(\mathrm{~s}, 3 \mathrm{H}), 2.549-2.911(\mathrm{q}, \mathrm{J}=7.56 \mathrm{~Hz}, 2 \mathrm{H}), 7.154-7.484(\mathrm{~m}, 4 \mathrm{H})$

p-Methoxyphenyl ethyl sulfoxide (Entry 10, Table 2)

IR $\left(\mathrm{CHCl}_{3}, \mathrm{~cm}^{-1}\right): v$ 3005.9, 1253.8, 1213.6, 1039. ${ }^{1} \mathrm{H}$ NMR: $\delta 0.941(\mathrm{t}, \mathrm{J}=7.32 \mathrm{~Hz}, 3 \mathrm{H})$, 2.235-2.691 (m, 2H), $3.766(\mathrm{~s}, 3 \mathrm{H}), 6.812(\mathrm{~d}, \mathrm{~J}=8.58 \mathrm{~Hz}, 2 \mathrm{H}), 7.317(\mathrm{~d}, \mathrm{~J}=8.58 \mathrm{~Hz}, 2 \mathrm{H})$. 
p-Chlorophenyl ethyl sulfoxide (Entry 11, Table 2)

IR $\left(\mathrm{CHCl}_{3}, \mathrm{~cm}^{-1}\right): v 1481,1042.1,833,751.6 .{ }^{1} \mathrm{H}$ NMR: $\delta 1.030(\mathrm{t}, \mathrm{J}=7.32 \mathrm{~Hz}, 3 \mathrm{H})$, 2.28-2.84 (m, 2H), $7.492(\mathrm{~m}, 4 \mathrm{H})$

p-Acetamidophenyl ethyl sulfoxide (Entry 12, Table 2)

IR $\left(\mathrm{CHCl}_{3}, \mathrm{~cm}^{-1}\right): v$ 3308.2, 3271.8, 3004.2, 1678.7, 1210.6, 1034.4, 748.7. ${ }^{1} \mathrm{H}$ NMR: $\delta$ $0.985(\mathrm{t}, \mathrm{J}=7.38 \mathrm{~Hz}, 3 \mathrm{H}), 2.105(\mathrm{~s}, 3 \mathrm{H}), 2.59-2.95(\mathrm{q}, \mathrm{J}=7.38 \mathrm{~Hz}, 2 \mathrm{H}), 7.211-7.757(\mathrm{~m}$, 4H), $8.746(\mathrm{~s}, 1 \mathrm{H})$.

\section{2-Cyanoethyl phenyl sulfoxide (Entry 13, Table 2)}

IR $\left(\mathrm{CHCl}_{3}, \mathrm{~cm}^{-1}\right): v$ 3017.8, 2256.5, 1223.4, 1045, 761.4. ${ }^{1} \mathrm{H}$ NMR: $\delta$ 2.700-3.054 (m, 4H), $7.537(\mathrm{~m}, 5 \mathrm{H})$.

\section{2-Ethoxycarbonylethyl phenyl sulfoxide (Entry 14, Table 2)}

IR $\left(\mathrm{CHCl}_{3}, \mathrm{~cm}^{-1}\right): v 3014.3,1730.7,1217.1,1037.8,745.1 .{ }^{1} \mathrm{H}$ NMR: $\delta 1.054(\mathrm{t}, \mathrm{J}=7.08$ $\mathrm{Hz}, 3 \mathrm{H}), 2.496-2.724(\mathrm{~m}, 2 \mathrm{H}), 2.878-3.249$ (m, 2H), 3.864-4.222 (q, J= 7.08 Hz, 2H), 7.378-7.647 (m, 5H).

\section{2-Methoxycarbonyl-1-methylethyl phenyl sulfoxide (Entry 15, Table 2)}

IR $\left(\mathrm{CHCl}_{3}, \mathrm{~cm}^{-1}\right): v$ 3030.5, 1713.2, 1217.1, 1039.6, 754.4. ${ }^{1} \mathrm{H}$ NMR: $\delta 1.55(\mathrm{~d}, \mathrm{~J}=6.84$ $\mathrm{Hz}, 3 \mathrm{H}), 2.71(\mathrm{t}, \mathrm{J}=7.32 \mathrm{~Hz}, 2 \mathrm{H}), 3.9-4.303(\mathrm{~m}, 4 \mathrm{H}), 7.47-7.68(\mathrm{~m}, 5 \mathrm{H})$

\section{2-Hydroxycarbonyl-1-methylethyl phenyl sulfoxide (Entry 16, Table 2)}

IR $\left(\mathrm{CHCl}_{3}, \mathrm{~cm}^{-1}\right): v 3034.6,1715.3,1076.9,742.5 .{ }^{1} \mathrm{H} \mathrm{NMR}: \delta 1.279(\mathrm{~d}, \mathrm{~J}=6.84 \mathrm{~Hz}$, 3H), 2.211-3.771 (m, 3H), 7.41-7.891 (m, 5H).

\section{3-Hydroxy-1-methylpropyl phenyl sulfoxide (Entry 17, Table 2)}

IR $\left(\mathrm{CHCl}_{3}, \mathrm{~cm}^{-1}\right): v$ 3393.9, 3004.2, 1216.8, 1028.2, 760.8. ${ }^{1} \mathrm{H}$ NMR: $\delta 0.989(\mathrm{~d}, \mathrm{~J}=6.84$ $\mathrm{Hz}, 3 \mathrm{H}), 1.637-2.325$ (m, 2H), 2.846-3.184 (m, 2H), $3.803(\mathrm{~m}, 2 \mathrm{H}), 7.529(\mathrm{~m}, 5 \mathrm{H})$. 


\section{3-Hydroxy-1-methylpropyl phenyl sulfoxide (Entry 18, Table 2)}

IR $\left(\mathrm{CHCl}_{3}, \mathrm{~cm}^{-1}\right): v 3387.2,3004.2,1216.8,1028.2,760.8 .{ }^{1} \mathrm{H}$ NMR: $\delta 0.883(\mathrm{~d}, \mathrm{~J}=6.84$ $\mathrm{Hz}, 3 \mathrm{H}), 1.65-2.41$ (m, 2H), 2.93-3.31 (m, 2H), 3.87 (m, 2H), 7.41-7.83 (m, 4H).

\section{Diphenyl sulfoxide (Entry 19, Table 2)}

m.p: $70-72^{\circ} \mathrm{C} . \mathrm{IR}\left(\mathrm{CHCl}_{3}, \mathrm{~cm}^{-1}\right): v$ 3005.1, 1033.7. ${ }^{1} \mathrm{H}$ NMR: $\delta$ 6.983-7.496 (m, 6H), 7.916-8.050 (m,4H)

\section{Benzyl phenyl sulfoxide (Entry 20, Table 2)}

m.p: $123-124^{\circ} \mathrm{C} . \mathrm{IR}\left(\mathrm{KBr}, \mathrm{cm}^{-1}\right): v$ 3004.4, 1028.6. ${ }^{1} \mathrm{H}$ NMR: $\delta 3.889(\mathrm{~s}, 2 \mathrm{H}), 6.959-$ 7.329 (m, 10H). MS (EI): $216\left(\mathrm{M}^{+}\right)$

\section{References:}

1. (a) Gilman, H.; Beaber, N. J. J. Am. Chem. Soc. 1925, 47, 1449. (b) Leandri, G.; Mangini, A.; Passerini, R. Gazz. Chim. Ital. 1954, 84, 3. (c) Herriott, A. W. Synthesis 1975, 447.

2. Burton, H.; Hu, P. F. J. Chem. Soc. 1948, 603.

3. (a) Gilman, H.; Beaber, N. J. J. Am. Chem. Soc. 1925, 47, 1449. (b) Bordwell, F. G.; Boutan, P. J. J. Am. Chem. Soc. 1957, 79, 717. (c) Xia, M.; Chen, Zc. Synth. Commun. 1997, 27(8), 1315.

4. Shriner, R. L.; Struck, H. C.; Jorison, W. J. J. Am. Chem. Soc. 1930, 52, 2060.

5. (a) Gasparrini, F.; Giovannoli, M.; Misti, D. J. Org. Chem. 1990, 55, 1323 and references cited therein. (b) Herriott, A. W.; Picker, D. Synthesis 1975, 447. (c) Baliah, V.; Uma, M. Tetrahedron 1963, 19, 455. (d) Srinivasan, C.; Jegatheesan, P. Pandarakutty; Arumugam, N. Indian J. Chem. Sect. A 1986, 25A, 678. (e) Drabowicz, J.; Dudzinski, B.; Mikolajczyk, M. Synlett, 1992, 3, 252. (f) Takeuchi, 
H.; Minato, H.; Kobayashi, M.; Yoshida, M.; Matsuyama, H.; Kamigata, N. Phosphorus Sulfur Silicon Relat. Elem. 1990, 47, 165. (g) Krishnan, G.; Sampson, P. Tetrahedron Lett., 1990, 31, 5609. (h) Breitschuh, R.; Seebach, D. Synthesis, 1992, 83. (i) Renaud, P.; Carrupt, P.; Gerster, M.; Schenk, K. Tetrahedron Lett. 1994, 35, 1703. 